\title{
Impedance-based damage detection under noise and vibration effects
}

\author{
Leandro M Campeiro, Ricardo ZM da Silveira and \\ Fabricio G Baptista
}

\begin{abstract}
The electro-mechanical impedance technique has been extensively studied in recent decades as a non-destructive method for detecting structural damage in structural health monitoring applications using low-cost piezoelectric transducers. Although many studies have reported the effectiveness of this detection method, numerous practical problems, such as the effects of noise and vibration, need to be addressed to enable this method's effective use in real applications. Therefore, this article presents an experimental analysis of noise and vibration effects on structural damage detection in impedance-based structural health monitoring systems. The experiments were performed on an aluminum bar using two piezoelectric diaphragms, where one diaphragm was used to measure the electrical impedance signatures and the other diaphragm was used as an actuator to generate noise and controlled vibration. The effects of noise and vibration on impedance signatures were evaluated by computing the coherence function and basic damage indices. The results indicate that vibration and noise significantly affect the threshold of the lowest detectable damage, which can be compensated by increasing the excitation signal of the piezoelectric transducer.
\end{abstract}

\section{Keywords}

Piezoelectric transducers, structural health monitoring, impedance, noise, vibration, damage

\section{Introduction}

Structural health monitoring (SHM) is a relevant field of research for the development of systems capable of monitoring and detecting structural damage in various types of engineering structures, thereby allowing efficient preventive maintenance and increasing the safety of the users of such structures. ${ }^{1,2}$ Areas of application include civil infrastructure, such as bridges, and large means of transport such as ships and aircraft. ${ }^{3}$

Several methods for damage identification, ${ }^{4}$ including data acquisition (DAQ), signal processing tools, and feature extraction, have been proposed. Damage detection should be based on a non-destructive testing (NDT) method, such as acoustic emission, ${ }^{5}$ Lamb waves, ${ }^{6}$ and fiber optic sensors, ${ }^{7}$ to be minimally invasive to the monitored structure. Among several methods, electro-mechanical impedance $(\mathrm{EMI})^{8-10}$ is an NDT technique known for using small and lightweight piezoelectric transducers, therein operating simultaneously as sensor and actuator.

The EMI method is based on the piezoelectric effect, ${ }^{11}$ which establishes a relation between the mechanical properties of the structure and the electrical properties of the transducer attached to it. Therefore, it is possible to monitor and analyze the integrity of the structure by measuring and analyzing the electrical impedance of the transducer, tasks that are simple to perform. The detection and quantification of damage are usually performed by comparing two electrical impedance signatures of each transducer, one of which is obtained when the structure is in a condition considered healthy and pre-stored as a reference, also called a baseline.

Although the EMI technique has been intensively studied in recent decades, many practical problems have limited its use in real applications. One of the most critical problems is the variations in the impedance signatures caused by changes in the operational and

Universidade Estadual Paulista (UNESP), Faculdade de Engenharia, Bauru, Brazil

\section{Corresponding author:}

Fabricio G Baptista, Departamento de Engenharia Elétrica, Faculdade de Engenharia, Universidade Estadual Paulista (UNESP), Bauru - SP I7033360, Brazil.

Email: fabriciogb@feb.unesp.br 
environmental conditions. As is well known, the correct diagnosis of the monitored structure under changing environments, ${ }^{12}$ such as temperature variations, ${ }^{13,14}$ vibration disturbances,,${ }^{14}$ and noise,${ }^{15}$ is critical in SHM applications, therein requiring advanced signal processing techniques ${ }^{16}$ for proper feature extraction.

Environmental changes are especially critical in impedance-based damage detection, which typically uses pre-stored data (baseline) as a reference to compute damage indices. Variations in the impedance signatures cause divergence from the pre-stored baseline, leading to a false-positive diagnosis of the monitored structure. To overcome this disadvantage, many researchers have proposed new signal processing tools ${ }^{17}$ for improved feature extraction and alternative methods that avoid the use of a pre-stored baseline, such as baseline-free ${ }^{18}$ and instantaneous baseline ${ }^{19}$ methods, although these methods typically require a set of transducers and cannot be applied under general monitoring conditions. Although the free-baseline concept is commonly used in the EMI method and in other methods $^{20}$ this statement is not entirely true, as is well known in one of the fundamental SHM axioms, ${ }^{21}$ making the investigation of the effects of environmental changes on damage detection an important field of research.

Therefore, in this article, we present an experimental investigation of the vibration and noise effects on the detection of structural damage in impedance-based SHM applications. Tests were performed on an aluminum bar on which two piezoelectric transducers were installed, one of the transducers being used to obtain the electrical impedance signatures and the other transducer being used for the generation of controlled vibrations and pseudorandom noise.

Different levels of vibration and noise were generated to evaluate the detection of damage under high and low signal-to-noise ratios (SNRs). The analysis of the SNR is fundamental in the EMI method because the piezoelectric transducers are typically capacitive, and consequently, their reactance and response voltage vary with the frequency, thus also varying the SNR. Commercial impedance analyzers utilize autobalanced bridges and other techniques to achieve impedance matching and keep the excitation and response signal constant. However, some low-cost alternative measurement systems, which are the focus of this study, do not keep the amplitude of the response signal of the transducer constant over wide frequency ranges, making the analysis of the vibration and noise effects relevant to the correct diagnosis of the monitored structure.

The principle of damage detection based on the EMI method is presented in the next section.

\section{EMI method}

\section{Principle}

The basic configuration of the EMI method consists of attaching a piezoelectric transducer to the surface of the structure to be monitored, as shown in Figure 1.

The transducer is connected to a DAQ device or measurement system that simultaneously excites the transducer and the structure while providing the electrical impedance signature $\left(Z_{E}(\omega)\right)$ of the transducer in an appropriate frequency range. Therefore, in the EMI method, the transducer operates simultaneously as sensor (direct piezoelectric effect) and actuator (reverse piezoelectric effect), and thus, a relationship is established between the mechanical impedance $\left(Z_{S}(\omega)\right)$ of the monitored structure and the electrical impedance $\left(Z_{E}(\omega)\right)$ of the transducer. Therefore, it is possible to monitor the health of the structure by measuring and analyzing the electrical impedance of the transducer.

The basic constitutive relations ${ }^{11}$ of the direct and reverse piezoelectric effects for a piezoelectric material are given by equations (1) and (2), respectively

$$
\begin{gathered}
D_{i}=d_{i k l} T_{k l}+\varepsilon_{i k}^{T} E_{k} \\
S_{i j}=s_{i j k l}^{E} T_{k l}+d_{k i j} E_{k}
\end{gathered}
$$

where $D_{i}$ and $E_{k}$ are the electrical displacement and the electric field components, respectively; $T_{k l}$ and $S_{i j}$ are the mechanical stress and strain components, respectively; $d_{i k l}, d_{k i j}, \varepsilon_{i k}^{T}$, and $s_{i j k l}^{E}$ are the piezoelectric, dielectric, and elastic compliance constants of the piezoelectric material, in which the superscripts $T$ and $E$ indicate constant stress and constant electric field, respectively, and the subscripts $i, j, k$, and $l$ take on the values of 1,2 , and 3 , representing the axes of the natural coordinate system of the piezoelectric material.

As indicated in equations (1) and (2), the electrical and mechanical properties characterizing piezoelectric materials are non-isotropic, and therefore, many twodimensional and three-dimensional models have been proposed to relate the electrical impedance of the transducer and the mechanical properties of the structure. ${ }^{22}$ However, the transducers typically used in the EMI method are small and thin PZT (lead zirconate titanate) ceramics, which make the deformations along the thickness minimal. In addition, if the monitored structure is small, as in the case of aluminum bars normally used in laboratories, a one-dimensional model as proposed by Liang et al. ${ }^{23}$ may be satisfactory at sufficiently low frequency. A simplified version of this model is given by

$$
Z_{E}(\omega)=\frac{1}{j \omega C}\left(1-\frac{d_{31}^{2}}{s_{11}^{E} \varepsilon_{33}^{T}} \frac{Z_{S}(\omega)}{Z_{S}(\omega)+Z_{P}(\omega)}\right)^{-1}
$$




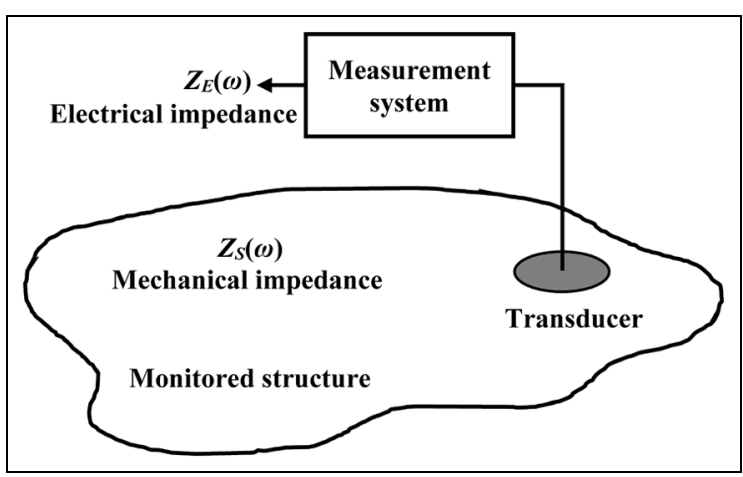

Figure I. Basic configuration of the EMI method.

where $Z_{E}(\omega)$ is the electrical impedance of the transducer at angular frequency $\omega$, which should vary within an appropriate range provided by the measuring system; $Z_{P}(\omega)$ is the mechanical impedance of the transducer; $Z_{S}(\omega)$ is the mechanical impedance of the monitored structure; $C$ is the static capacitance of the transducer; $d_{31}, s_{11}^{E}$, and $\varepsilon_{33}^{T}$ are the properties of the piezoelectric material as defined above but considering a one-dimensional assumption; and $j$ is the unit imaginary number.

According to equation (3), any variation in the mechanical impedance of the structure $\left(Z_{S}(\omega)\right)$ due to damage causes a corresponding variation in the electrical impedance of the transducer $\left(Z_{E}(\omega)\right)$. Therefore, structural damage can be detected by measuring and analyzing the electrical impedance signatures of the transducer. This analysis is usually performed by comparing two impedance signatures using damage indices, as shown in the next section.

\section{Damage indices}

As mentioned above, structural damage detection is typically performed by comparing two impedance signatures, where one signature is obtained when the structure is in a state considered healthy and pre-stored as a baseline. This quantitative comparison is performed using damage indices. Two basic damage indices are widely used in the literature: the root-mean-square deviation (RMSD) and the correlation coefficient deviation metric (CCDM).

The RMSD index is based on the Euclidean norm, which can be calculated in multiple ways. In this study, the $R M S D$ index was calculated as ${ }^{2}$

$$
R M S D=\sum_{\omega=\omega_{I}}^{\omega_{F}} \sqrt{\frac{\left[Z_{E, 2}(\omega)-Z_{E, 1}(\omega)\right]^{2}}{\left[Z_{E, 1}(\omega)\right]^{2}}}
$$

where $Z_{E, 1}(\omega)$ is the baseline signature, $Z_{E, 2}(\omega)$ is the impedance signature after possible damage, and $R M S D$ is the index calculated in the frequency range with initial frequency $\omega_{I}$ and final frequency $\omega_{F}$. The impedance signatures are complex, and the index can be calculated using the real part, imaginary part, or magnitude of the impedance signatures.

The $C C D M$ index is based on the correlation coefficient and is given $b y^{2}$

$$
C C D M=1-C_{C}
$$

where $-1<C_{C}<1$ is the correlation coefficient computed as

$$
C_{C}=\frac{\operatorname{cov}\left[Z_{E, 1}(\omega), Z_{E, 2}(\omega)\right]}{\sigma_{1} \sigma_{2}}
$$

in which "cov" is the covariance of the two impedance signatures, as previously defined, calculated in an appropriate frequency range $\left(\omega_{I}-\omega_{F}\right)$, and $\sigma_{1}$ and $\sigma_{2}$ are the corresponding standard deviations of each signature. Similar to the RMSD index, the CCDM index can be calculated using the real part, imaginary part, or magnitude of the impedance signatures.

Since the damage detection is based on the comparison between two impedance signatures, the measurement system must have good accuracy to provide good repetition between the impedance signatures. The measurement systems applied in the EMI technique are discussed in the next section.

\section{Measurement system}

Traditionally, the measurement of impedance signatures has been performed predominantly by commercial impedance analyzers such as HP 4194A and HP 4294A. Although these instruments are being gradually replaced by alternative measurement systems, they have been used in recent studies ${ }^{24,25}$ on the EMI method due to these systems' high accuracy.

Despite the high accuracy and precision of conventional impedance analyzers, these instruments are expensive, have many features that are not required for the EMI method, and are not suitable for field applications. Therefore, many researchers have proposed alternative low-cost measurement systems, such as those based on the commercial chip AD5933, ${ }^{26}$ and other more versatile solutions. ${ }^{27-29}$ In this study, we used an alternative measurement system $^{28}$ based on a DAQ device and a personal computer (PC) for the signal processing. The system has been modified to generate vibration and controlled noise while measuring the impedance signatures. The system is shown in Figure 2.

According to Figure 2, the impedance measurement is based on the voltage divider constituted by the series resistor $\left(R_{S}\right)$ and the piezoelectric transducer with electrical impedance $Z_{E}(\omega)$. The excitation signal $(x(t))$ is 
generated by the analog output $\left(\mathrm{AO}_{0}\right)$ from the digitalto-analog converter (DAC), and the transducer response signal $(y(t))$ is sampled by the analog input $\left(\mathrm{AI}_{0}\right)$ from the analog-to-digital converter (ADC). The signals $x[n]$ and $y[n]$ are the excitation and response signals, respectively, in discrete time. To ensure good reproducibility of the impedance measurements, the response signal $(y(t))$ from the transducer should be sampled synchronously with the generation of the excitation signal $(x(t))$.

Considering that the impedance of the analog input is sufficiently high to be disregarded, it can be demonstrated that the electrical impedance of the transducer $\left(Z_{E}(\omega)\right)$ is given by ${ }^{28}$

$$
Z_{E}(\omega)=R_{S} \frac{H(\omega)}{1-H(\omega)}
$$

where $H(\omega)$ is the frequency response function (FRF), taking the excitation signal $(x[n])$ as input and the response signal $(y[n])$ as output.

This system based on the voltage divider is easy to implement, and similar solutions have been used in several studies. ${ }^{27,29}$ However, this system has the disadvantage of being unable to keep the amplitude of the response signal $(y(t))$ from the transducer constant; the amplitude of the response signal decreases with an increase in the frequency. Although, from a strictly electrical point of view, the impedance given by equation (7) does not depend on the signal amplitude, lowvoltage signals can reduce the sensitivity to structural damage, especially in large structures. In addition, a low-voltage signal reduces the SNR, which can be critical in noisy environments.

In this study, the impedance signatures were obtained under high and low SNR, generating controlled noise and vibration, as detailed in the next section.

\section{External vibration and noise}

To evaluate the effects of external vibration and noise on the detection of structural damage, a piezoelectric transducer operating as an actuator was excited using an analog output $\left(\mathrm{AO}_{1}\right)$ of the $\mathrm{DAQ}$, as shown in Figure 2. Since commercial devices typically have an output current drive on the order of a few milliamps, an operational amplifier used as a buffer may be necessary for proper excitation of the actuator, as indicated. The signals $w(t)$ and $w[n]$ are the vibration and noise signals generated in continuous and discrete time, respectively.

The term vibration is used to describe the motion of a body with respect to a reference point, which can be of various types involving tiny air particles or

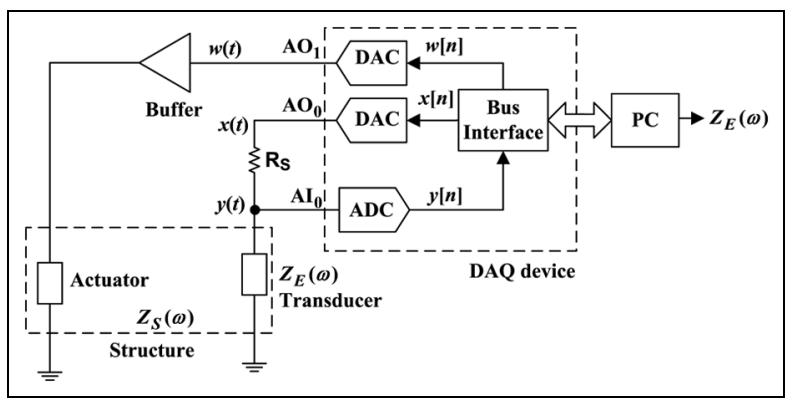

Figure 2. System used for impedance measurement and generation of vibration and noise.

structures. ${ }^{30}$ In practice, the word vibration is typically used to describe motions in structures, such as machinery, bridges, and aircraft, which are of interest for SHM applications.

An important type of vibration is produced by rotating devices. Rotational speeds are usually in the range of $60-120,000 \mathrm{r} / \mathrm{min}$, which correspond to mechanical vibrations in the frequency range of $1 \mathrm{~Hz}-2 \mathrm{kHz} .{ }^{30} \mathrm{To}$ evaluate the effects of such vibrations on impedancebased damage detection, the piezoelectric actuator was excited by a linear chirp signal in a similar frequency range. The chirp signal is given by

$$
w[n]=A \sin \left(\frac{2 \pi}{F_{S}} n\left(\frac{f_{2}-f_{1}}{2 N} n+f_{1}\right)\right)
$$

where $f_{1}$ and $f_{2}$ are the initial and final frequency, respectively, $n$ is the sample, $N$ is the number of samples, $F_{S}$ is the sample rate, and $A$ is the amplitude of the signal.

The frequencies of the chirp signal were defined as $f_{1}=1 \mathrm{~Hz}$ and $f_{2}=2 \mathrm{kHz}$ to generate vibrations similar to rotating devices. In addition, the other parameters were defined as $F_{S}=2 \mathrm{MS} / \mathrm{s}, N=1 \mathrm{M}$, and an amplitude $A$ ranging from $0.5 \mathrm{~V}(1 \mathrm{Vpp})$ to $25 \mathrm{~V}$ $(50 \mathrm{Vpp})$ to obtain different vibration intensities. As an example, a chirp signal with amplitude of $1 \mathrm{~V}$ and the corresponding power spectral density (PSD) are shown in Figure 3.

As seen in Figure 3, the spectrum of the chirp signal contains frequencies in the range from approximately $1 \mathrm{~Hz}$ to $2 \mathrm{kHz}$, similar to the vibrations caused by rotating devices, thereby allowing one to analyze the effects of vibrations of this type on the detection of structural damage.

Unlike the vibrations described above, noise is generally a random signal and does not have well-defined frequency components, occurring in any range of the spectrum. Random noise can be generated by various types of sources such as ventilation systems, jets, blowers, combustion chambers, automobile ignition, 


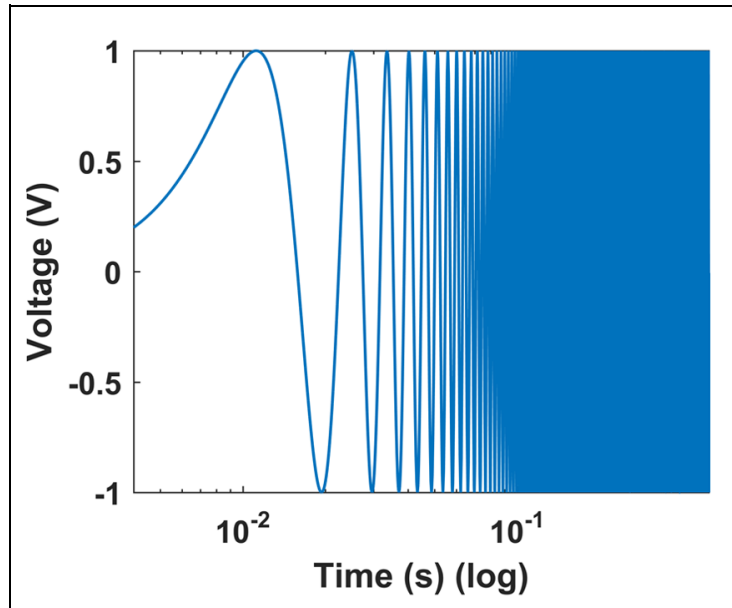

(a)

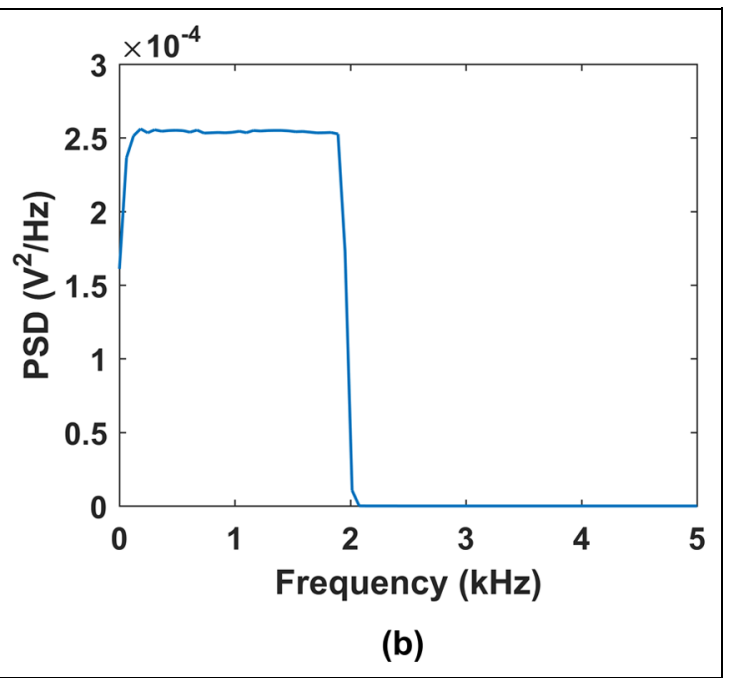

Figure 3. (a) Chirp signal and (b) the corresponding PSD.

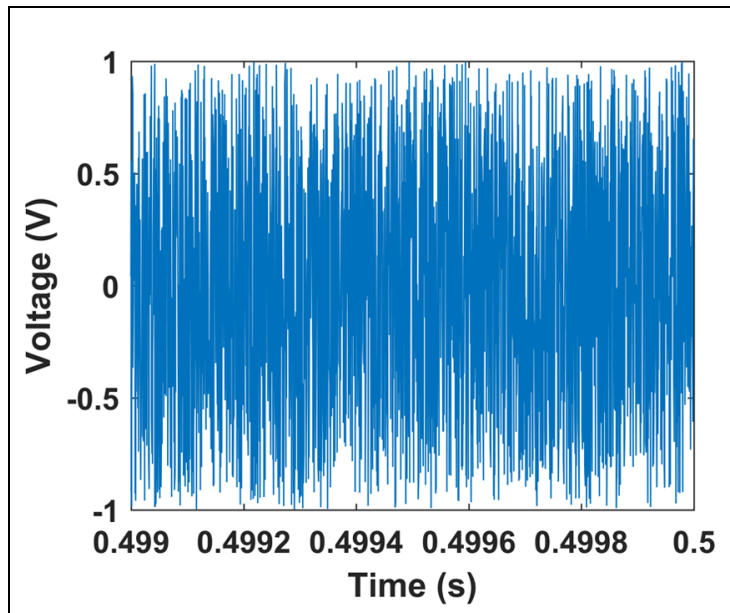

(a)

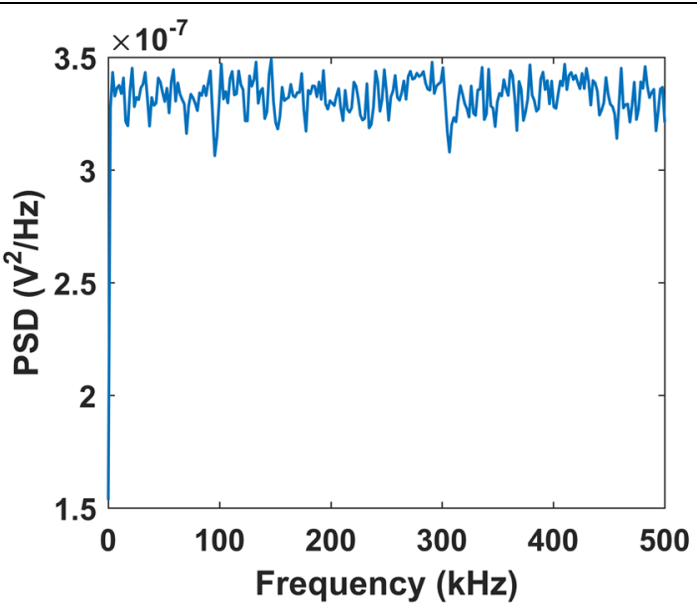

(b)

Figure 4. (a) White noise signal and (b) the corresponding PSD.

fluorescent lights, lighting, and solar radiation. Random noise can also be generated by piezoelectric transducers, conductors, and measuring systems due to thermal motion and other causes.

A well-known type of noise is white noise, which presents a uniform frequency spectrum over a wide range. In this study, a pseudorandom white noise was generated in the range of $0-500 \mathrm{kHz}$ and with amplitude ranging from $0.5 \mathrm{~V}(1 \mathrm{Vpp})$ to $25 \mathrm{~V}(50 \mathrm{Vpp})$ to analyze the influence of noise of different intensities on the detection of damage. White noise with amplitude of $1 \mathrm{~V}$ and the corresponding PSD are shown in Figure 4.

According to Figure 4, the white noise used in this study has an approximately uniform spectrum in the frequency range from 0 to $500 \mathrm{kHz}$, which is the same range in which the impedance signatures were obtained, allowing one to analyze the noise effects on the detection of damage. Notably, the uniform spectrum shown in Figure 4 is not typically obtained by exciting the structure. The effective excitation of the host structure depends considerably on the electro-mechanical coupling of the piezoelectric transducer, the characteristics of the host structure, distance from the transducer, and the propagation of the waves in the structure that have frequency-dependent attenuation. Therefore, the noise generation may be less efficient at some frequency bands, particularly for large structures. However, as described in the next section, a small aluminum bar was used in the experimental tests which enables satisfactory excitation across the spectrum. 


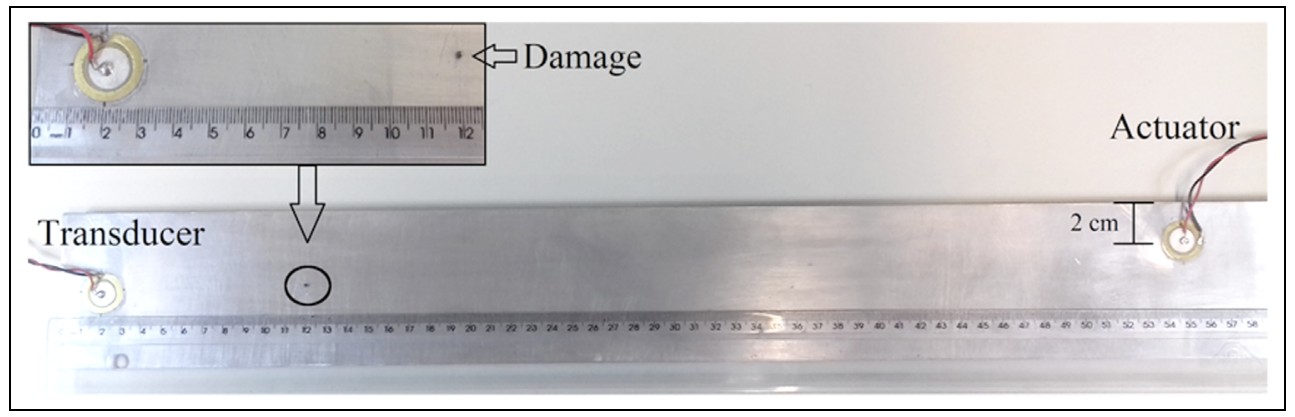

Figure 5. Aluminum bar with the two transducers installed; in detail, the inducing of damage by adding a small metal ball.

The effects of vibration and noise on the impedance signatures can be quantitatively evaluated by computing the coherence function between the excitation signal $(x[n])$ and the response signal $(y[n])$ from the transducer, as shown in Figure 2. The coherence function was calculated as

$$
C_{x y}(\omega)=\frac{\left|S_{x y}(\omega)\right|^{2}}{S_{x x}(\omega) S_{y y}(\omega)}
$$

where $C_{x y}(\omega)$ is the magnitude-squared coherence, $S_{x x}(\omega)$ and $S_{y y}(\omega)$ are the PSD estimates of the excitation and the response signal, respectively, and $S_{x y}(\omega)$ is the crossed PSD.

In an ideal condition, the excitation and response signals are fully coherent, and therefore, $C_{x y}(\omega)=1$ across the frequency range. In contrast, if the signals are contaminated by noise or vibration, the coherence tends to decrease in the frequency bands where noise and vibration occur.

The next section presents the experimental setup used to evaluate the vibration and noise effects.

\section{Experimental setup}

To evaluate the effects of external vibration and noise on the damage detection based on the EMI method, experiments were performed on an aluminum bar with dimensions of $110 \mathrm{~mm} \times 76 \mathrm{~mm} \times 3 \mathrm{~mm}$ and mass of approximately $664 \mathrm{~g}$. The transducers used in this study are piezoelectric diaphragms, ${ }^{31}$ which have characteristics and behavior similar to conventional PZT ceramics. The piezoelectric diaphragms consist of a circular brass plate with dimensions of $20 \mathrm{~mm} \times 0.20 \mathrm{~mm}$, on which a circular piezoelectric ceramic with dimensions of $14 \mathrm{~mm} \times 0.22 \mathrm{~mm}$ is mounted.

Two transducers were used according to the configuration shown in Figure 2: one transducer was used for impedance measurements, and the other transducer was used as an actuator for the generation of noise and vibration.
Figure 5 shows the specimen with the two transducers installed and the dimensions and distances used in the experiments. In addition, the details show the structural damage induced in the structure by adding the metal mass.

The transducers were fixed to the structure using cyanoacrylate glue, and the specimen was supported on the workbench by rubber blocks. The measurement of electrical impedance signatures and the generation of vibration and noise were performed using the NI USB$6366 \mathrm{DAQ}$ device with a sampling rate of $2 \mathrm{MS} / \mathrm{s}$. The impedance signatures were obtained in the frequency range of $0-500 \mathrm{kHz}$ with a step of $2 \mathrm{~Hz}$. Notably, although the used rate of $2 \mathrm{MS} / \mathrm{s}$ satisfies the Nyquist sampling theorem, the accuracy and precision of the impedance measurement decrease for frequencies higher than $200 \mathrm{kHz}$ for the DAQ device used in this work. However, the variations in impedance signatures caused by the measurement system are very low and therefore negligible compared with the external vibration and noise considered in this study.

The excitation signal $(x[n])$ of the impedance measurement system is a linear chirp signal, which was initially set to a $1-\mathrm{V}$ amplitude; later, higher levels were tested to compensate the noise effects. The transducer was connected to the DAQ device as shown in Figure 2 using a series resistor $\left(R_{S}\right)$ of $2.2 \mathrm{k} \Omega$, which is suitable for the current drive and voltage range limits of the DAQ device and allows different SNR levels to be obtained.

Controlled vibration and pseudorandom noise with amplitude ranging from $0.5 \mathrm{~V}(1 \mathrm{Vpp})$ to $25 \mathrm{~V}$ $(50 \mathrm{Vpp})$ were generated following the procedure described above. Because the analog output of the DAQ device has low current drive and limited voltage range, an LM675 operational amplifier was used to achieve proper excitation of the piezoelectric actuator for noise and vibration generation.

The effects of vibration and noise were analyzed by computing the coherence function between the excitation and response signals and by analyzing the impedance signatures qualitatively and quantitatively by computing the RMSD and CCDM indices for the 


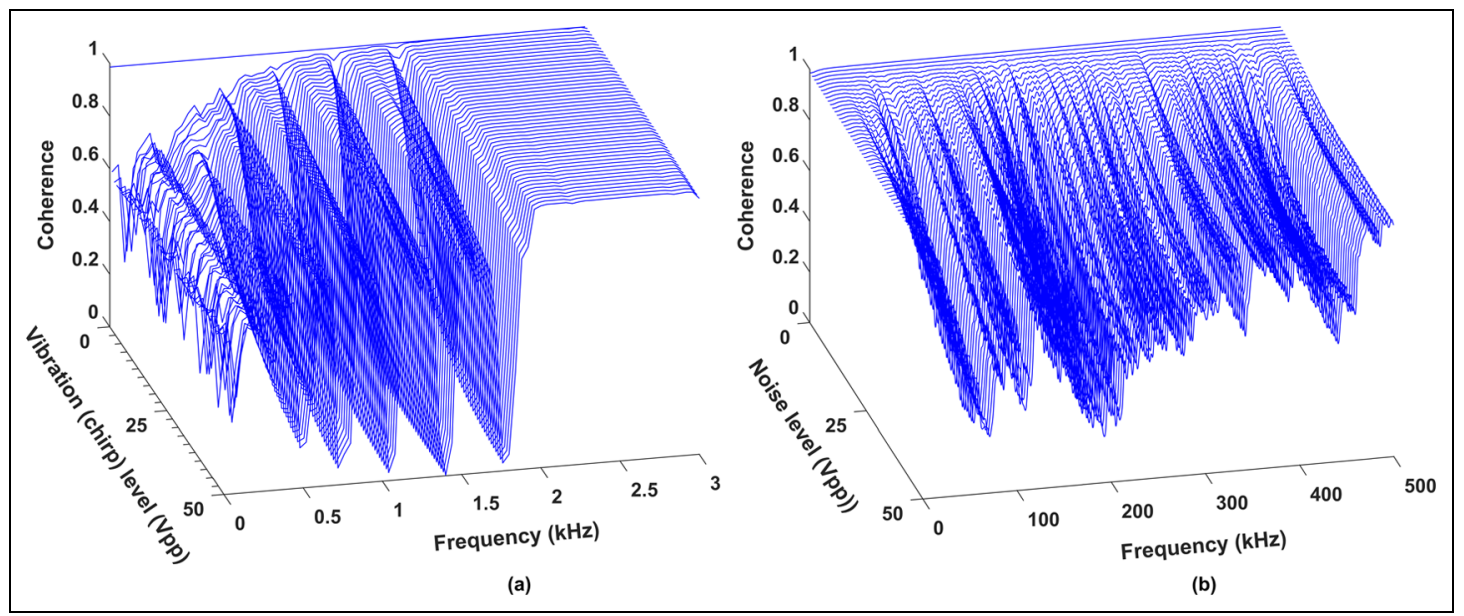

Figure 6. Coherence function obtained under (a) external vibration generated by a chirp signal and (b) white noise of different levels.

specimen under healthy and damaged conditions. Structural damage was induced in the specimen by attaching a small steel ball approximately $1 \mathrm{~mm}$ in diameter and with a mass of $15 \mathrm{mg}$, which is approximately $0.002 \%$ of the mass of the host structure, at a distance of $100 \mathrm{~mm}$ from the center of the transducer using cyanoacrylate glue, as detailed in Figure 5. Adding mass alters the mechanical impedance of the structure, which may be related to structural damage. This procedure has the advantage of not causing permanent damage to the specimen.

All measurements were performed at a temperature controlled by an air conditioner to mitigate the effects of temperature on the impedance signatures and by taking an average of five measurements under all conditions of vibration and noise. The experimental results are presented and discussed in the next section.

\section{Results and discussion}

\section{Coherence function}

As stated above, the coherence function is an important tool for indicating whether a signal is contaminated by external disturbances such as noise or vibration. In the measurement system shown in Figure 2, the coherence between the excitation $(x[n])$ and response $(y[n])$ signals under an ideal condition is expected to be of value 1 over the entire frequency range, indicating the linearity of the system and the absence of external disturbances.

Figure 6(a) shows the coherence function calculated using equation (9) obtained under the effects of external vibrations generated by the chirp signal given by equation (8) and shown in Figure 3 with different intensities.

As indicated in Figure 6(a), the vibration was generated by a chirp signal ranging from $0 \mathrm{~V}$ (no external vibration) to $50 \mathrm{Vpp}$, with steps of $1 \mathrm{Vpp}$. For the condition of no external vibration $(0 \mathrm{~V})$, there is only the noise floor inherently caused by the measurement system and other uncontrolled sources. Under this condition, the coherence is very close to 1 throughout the frequency range, indicating the linearity of the system and the high correlation between the excitation and response signals.

In contrast, as the vibration generated by the chirp signal increases, the coherence function decreases in the range of approximately $1 \mathrm{~Hz}-2 \mathrm{kHz}$, which is the same frequency range of the vibration. As shown in Figure 6(a), a vibration generated by a chirp signal of only $1 \mathrm{Vpp}$ is sufficient to abruptly reduce the coherence, particularly at low frequencies and up to approximately $500 \mathrm{~Hz}$. For higher frequencies, the coherence changes more gradually with the intensity of the vibration, registering at approximately 0 at some frequencies for a vibration intensity of $50 \mathrm{Vpp}$, thus indicating a weak correlation between the excitation and response signals. Under this condition, the measurement of the electrical impedance of the transducer may be inconsistent, and consequently, the detection of structural damage may be infeasible. For frequencies above $3 \mathrm{kHz}$, significant changes are not observed in the coherence functions, and the results are not shown in the figure.

The results obtained for the white noise of different intensities generated as shown in Figure 4 are shown in Figure 6(b). As observed in Figure 6(b), the coherence function decreases over the entire frequency range of $0-500 \mathrm{kHz}$ as the intensity of the white noise increases. In contrast to vibrations that normally occur in welldefined frequency bands, random noise can occur in any range of the spectrum and may hinder the selection of appropriate frequency bands for the calculation of damage indices in noisy environments. 


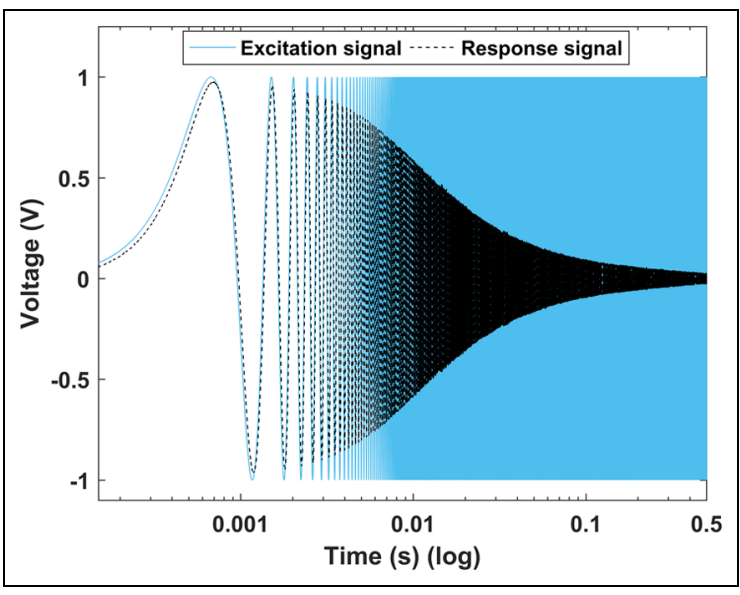

Figure 7. Comparison between the excitation and response signals.

As discussed above, it is important to note that the amplitude of the response signal $(y(t))$ from the transducer is not constant; rather, it decreases with an increase in the frequency due to the voltage divider consisting of the series resistor and the transducer, as shown in Figure 2. The comparison between the excitation signal with amplitude of $1 \mathrm{~V}$ and the corresponding response signal is shown in Figure 7.

As seen in Figure 7, the response signal decreases from a maximum value of approximately $2 \mathrm{Vpp}$ to a minimum value of approximately $0.04 \mathrm{Vpp}$. Consequently, the SNR is also not constant, ranging from approximately $6 \mathrm{~dB}$ for low frequencies and external noise of $1 \mathrm{Vpp}$ to approximately $-62 \mathrm{~dB}$ for a frequency of $500 \mathrm{kHz}$ and external noise of $50 \mathrm{Vpp}$. Under ideal conditions where there is only a noise floor of approximately $2 \mathrm{mVpp}$, the SNR is approximately $60 \mathrm{~dB}$ at low frequencies and $26 \mathrm{~dB}$ at high frequencies. However, according to the experimental results, the low level of the response signal at high frequencies has no significant impact on the coherence functions; as seen in Figure 6(b), the coherence is higher at high frequencies of approximately $500 \mathrm{kHz}$ than at low frequencies of approximately 200 and $50 \mathrm{kHz}$.

The effects of vibration and noise on the impedance signatures and damage indices are discussed in the next section.

\section{Impedance signatures and damage indices}

Although the low-frequency vibration generated by the chirp signal does not cause observable changes in the coherence function at higher frequencies as discussed in the previous section, a different result is observed in the electrical impedance signatures. As an example, Figure 8 shows the comparison of two impedance signatures (real part) corresponding to a resonance peak

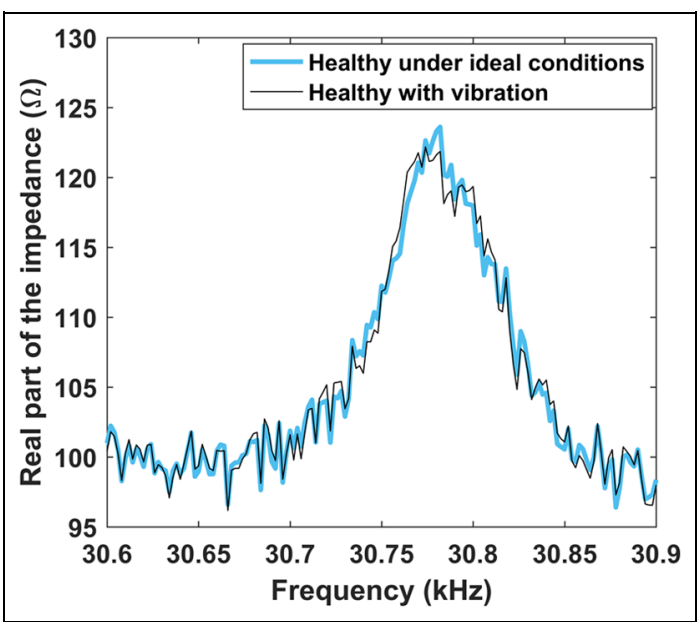

Figure 8. Effects of low-frequency vibrations on the impedance signatures at higher frequencies.

at approximately $30.78 \mathrm{kHz}$ obtained for the healthy structure under the conditions of no external disturbance and with vibration generated by the chirp signal of $50 \mathrm{Vpp}$.

As observed in Figure 8, changes due to vibration are observed in the impedance signature at approximately $30 \mathrm{kHz}$, although the vibration generated by the chirp signal is of low frequency, from $1 \mathrm{~Hz}$ to $2 \mathrm{kHz}$. Consequently, the damage indices will also be changed, which may make it difficult to detect incipient damages.

In this study, we used the RMSD and CCDM indices because they are widely reported in the literature to compare directly two impedance signatures, based on the Euclidean norm and correlation coefficient, respectively, when one of them is considered the baseline, thus allowing a quantitative evaluation of the variations in impedance signatures due to external vibrations and noise, such as the variations shown in Figure 8. As mentioned in section "Introduction," although advanced techniques for feature extraction that do not use a baseline signature have been proposed, the use of a reference is inevitable because of the fundamental SHM axioms. ${ }^{21}$ Therefore, quantitatively analyzing variations in the impedance signatures caused by external disturbances using basic indices, although there are other more advanced feature extraction tools, is relevant for future studies and for developing new signal processing techniques and damage indices.

To analyze the effects of the vibration on the damage indices across the entire frequency range, the RMSD and CCDM indices were calculated using equations (4) and (5), respectively, in sub-bands of $10 \mathrm{kHz}$. The vibration was generated by the chirp signal with a frequency range of $1 \mathrm{~Hz}-2 \mathrm{kHz}$ and an intensity ranging from 1 to $50 \mathrm{Vpp}$. 


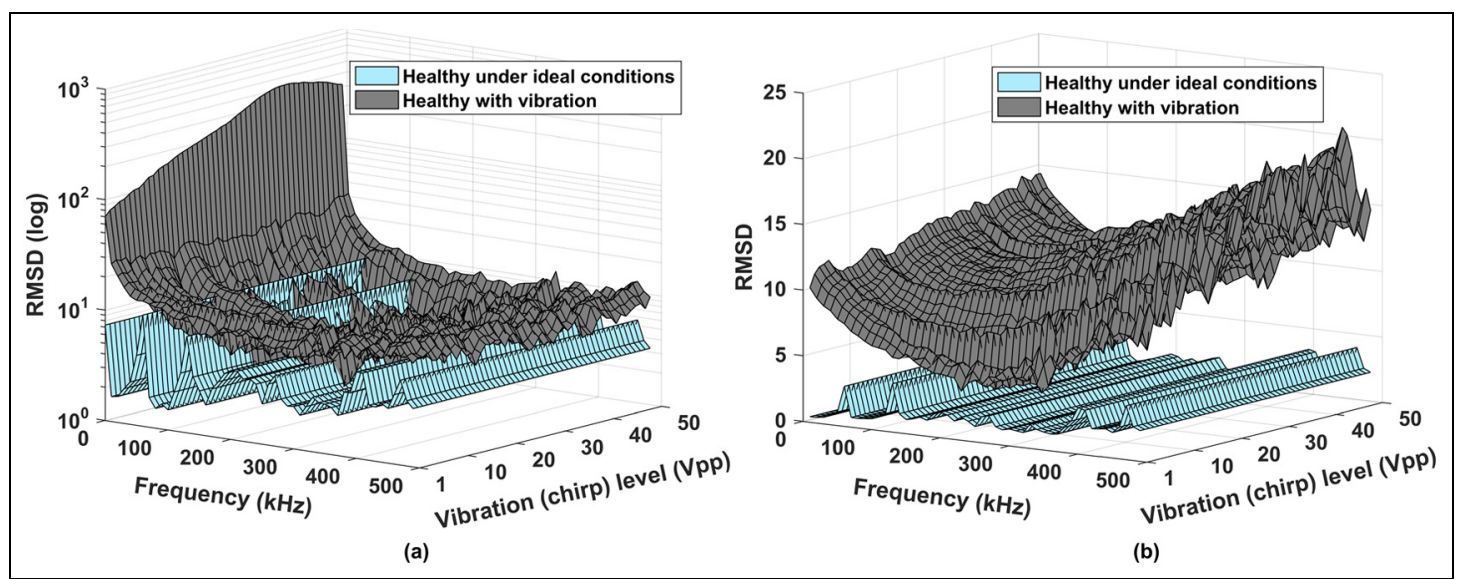

Figure 9. Effects of the low-frequency vibration on the RMSD index calculated using (a) the real part and (b) the imaginary part of the impedance.
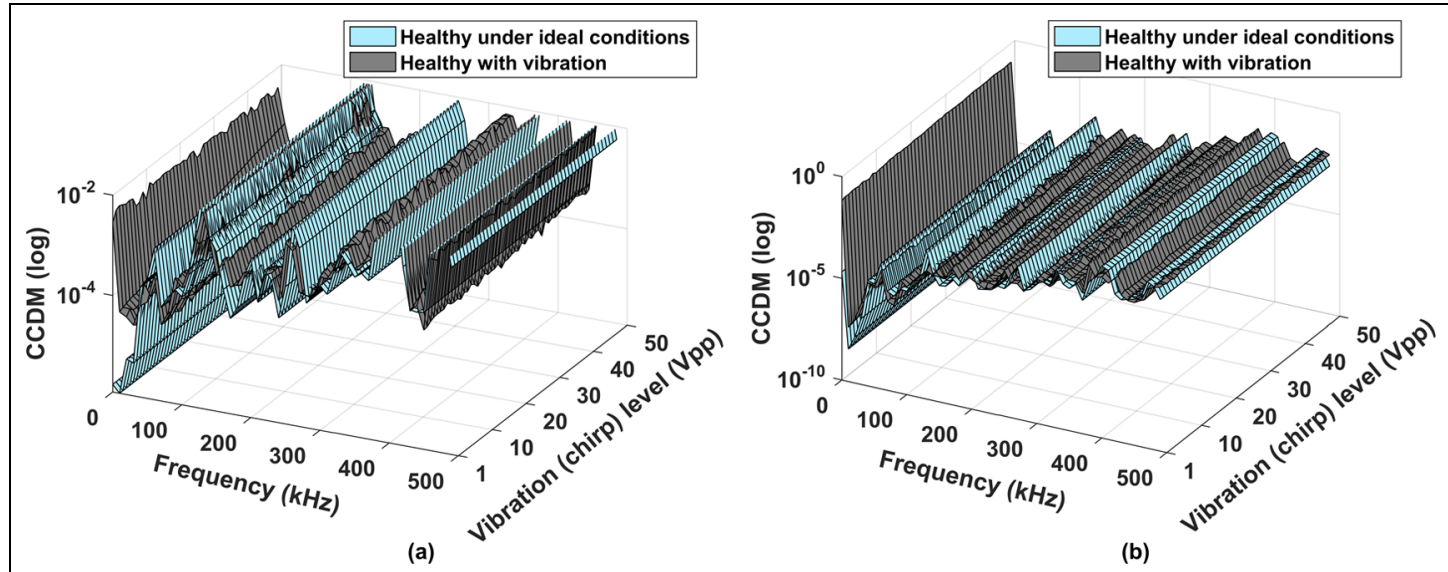

Figure 10. Effects of the low-frequency vibrations on the CCDM index calculated using (a) the real part and (b) the imaginary part of the impedance.

Figure 9 shows the results obtained for the RMSD index computed using the real and imaginary parts of the electrical impedance.

All RMSD indices were obtained for the healthy structure (i.e. without the addition of mass). The indices obtained under ideal conditions were computed using two impedance signatures measured without disturbance generation. The indices obtained with vibrations were computed using one of the signatures measured under ideal conditions and the corresponding impedance signature measured for each level of vibration. This procedure was also used to obtain the results shown in Figures 10, 12, and 13.

Because the structure is healthy, low damage indices are expected, given that under ideal conditions there are only slight variations in the impedance signatures caused by the noise floor, imprecisions in the measurement system, and other uncontrolled sources. However, as observed in Figure 9, the vibration increases the RMSD indices even for the healthy structure, which may make it difficult to define a threshold for the detection of incipient damage. For the indices calculated using the real part of the impedance, the variation is greater than $3000 \%$ at low frequencies and under intense vibration $(50 \mathrm{Vpp})$. Although the vibration is of low frequency, variations of greater than $150 \%$ in the indices are also observed at high frequencies. Variations are also observed throughout the frequency range in the indices calculated using the imaginary part of the impedance, but the variations are more significant at high frequencies.

The variations in the CCDM indices due to the lowfrequency vibrations are shown in Figure 10.

A different behavior is observed in the CCDM indices. Variations are observed only at low frequencies up to approximately $20 \mathrm{kHz}$. For higher frequencies, 


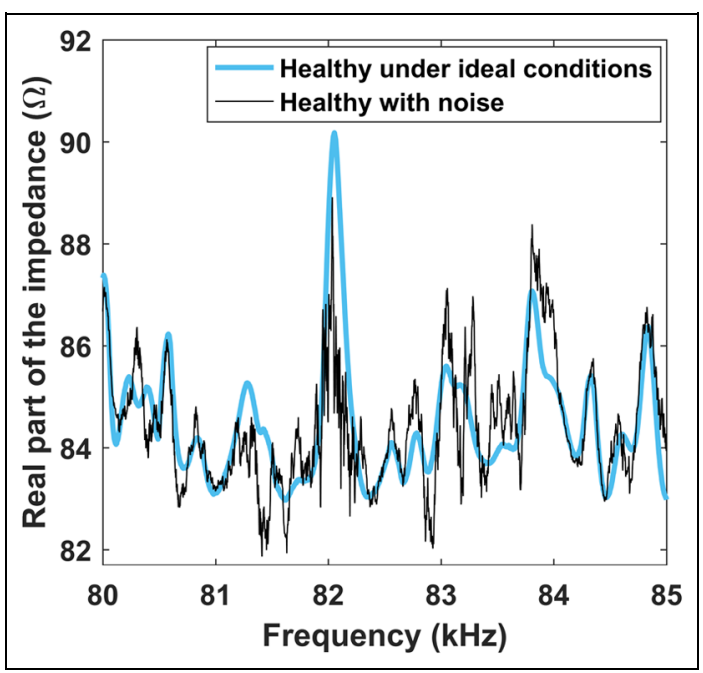

Figure II. Effects of the white noise on the impedance signatures.

the indices obtained under vibration and without any vibration take on approximately the same values.

A similar analysis was performed considering the white noise generated over the entire frequency spectrum of $0-500 \mathrm{kHz}$, as previously described. The effects of noise on impedance signatures are observed across the frequency range, even for low-intensity noise. As an example, Figure 11 shows the variations in the impedance signatures in the range of $80-85 \mathrm{kHz}$ caused by white noise with an amplitude of $1 \mathrm{~V}$.

A quantitative analysis of the noise effects is obtained by calculating the damage indices. The RMSD indices calculated in sub-bands of $10 \mathrm{kHz}$ using the real part and the imaginary part of the impedance and for different noise intensities are shown in Figure 12.

The noise effects were similar for the real part and the imaginary part of the impedance, as evidenced by the RMSD indices shown in Figure 12. The variations in the indices caused by the noise are significant across the analyzed frequency range since the white noise acts on the whole spectrum. The variations in the indices are approximately $600 \%$ even for noise of only $1 \mathrm{Vpp}$. For noise of $50 \mathrm{Vpp}$, the variations in the indices exceed $25,000 \%$. Notably, however, in most practical applications, the noise at high frequencies is typically less intense than that considered in this study; thus, less significant variations in the indices are expected. However, the analysis of high-intensity noise effects at high frequencies may be important for some specific applications where the monitored structure operates under very noisy environment. Another important observation is that the variation in the SNR caused by the measurement system does not significantly affect the results.

The results obtained for the CCDM indices under the influence of the white noise are shown in Figure 13.

According to Figure 13, the white noise also causes significant variations in the CCDM indices over the entire frequency range. For strong noise above $25 \mathrm{Vpp}$, the indices are close to 1, indicating that the correlation coefficient between the impedance signatures is close to 0 , as shown in equations (5) and (6). In addition, the indices increase with an increase in the frequency, most likely due to the variation in the SNR, which is lower at high frequencies.

Variations in indices caused by external vibration and noise make it difficult to set a threshold for damage detection, as discussed in the next section.

\section{Damage detection}

To analyze the feasibility of detecting incipient damage under the effects of vibration and noise, a small amount

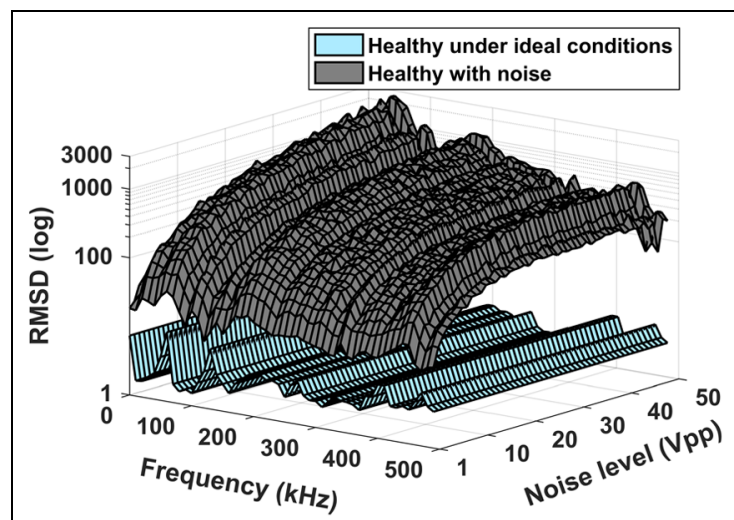

(a)

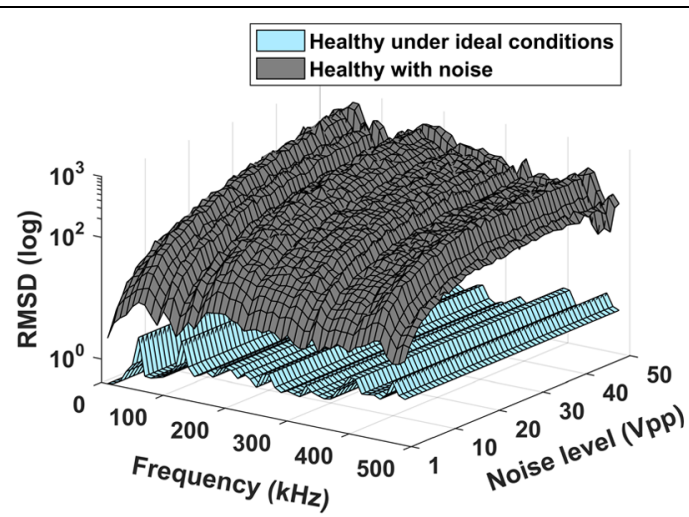

(b)

Figure 12. Effects of the white noise on the RMSD index calculated using (a) the real part and (b) the imaginary part of the impedance. 


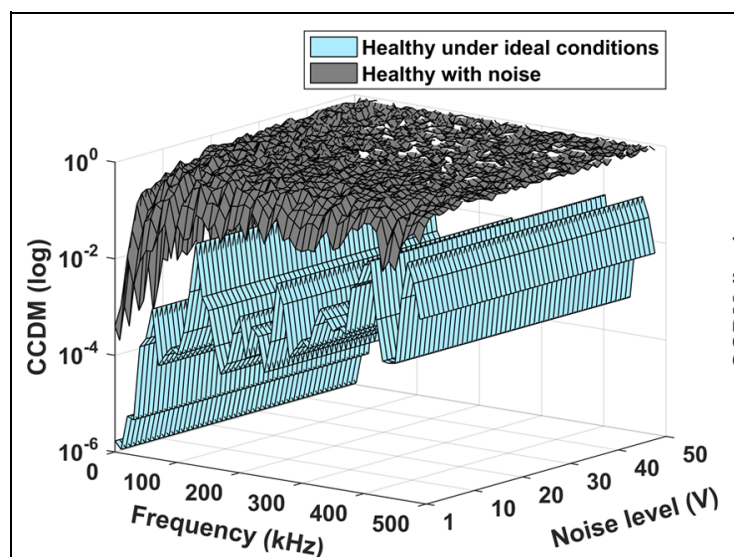

(a)

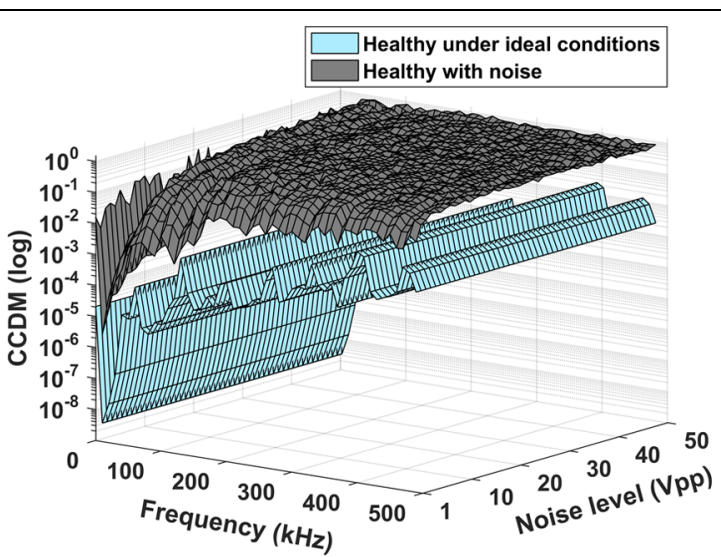

(b)

Figure 13. Effects of the white noise on the CCDM index calculated using (a) the real part and (b) the imaginary part of the impedance.

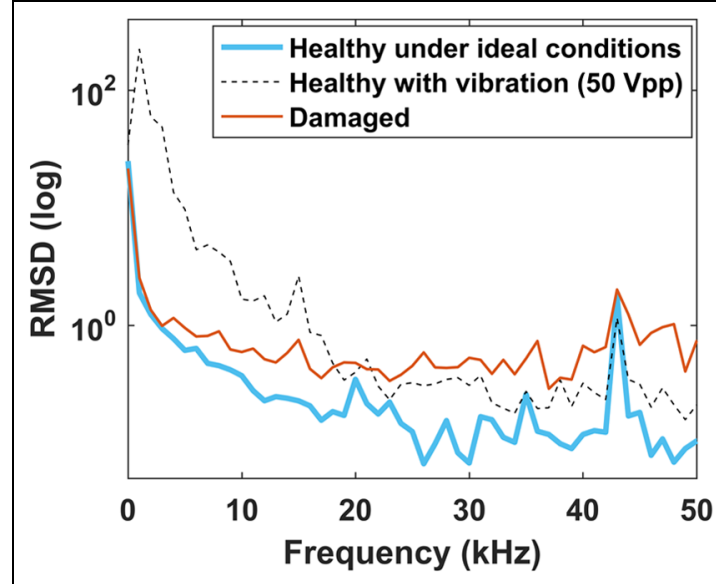

(a)

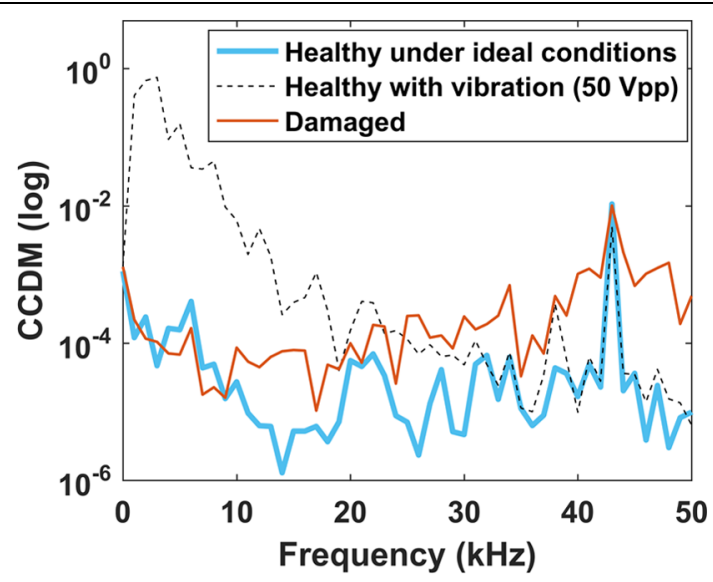

(b)

Figure I4. (a) RMSD and (b) CCDM indices obtained for ideal conditions, under low-frequency vibration, and for the damaged condition.

of damage was induced in the specimen following the procedure shown in Figure 5. The small steel ball causes a mass loading of only approximately $0.002 \%$ in the specimen, which represents a small amount of structural damage.

Figure 14 shows the RMSD and CCDM indices obtained for ideal conditions (no disturbance), under the effects of low-frequency vibration $(1 \mathrm{~Hz}-2 \mathrm{kHz})$ generated by a chirp signal of $50 \mathrm{Vpp}$, and for the damaged condition. The indices were calculated in subbands of $1 \mathrm{kHz}$.

The indices were computed by considering an impedance signature obtained without generating a vibration as the baseline. Two such impedance signatures were used to compute the indices obtained under ideal conditions, as shown in Figure 14.
Although the vibration is of low frequency up to $2 \mathrm{kHz}$, significant variations are observed in both indices at higher frequencies. The variations in the indices caused by the vibration are higher than the variations caused by the induced damage up to a frequency of approximately $20 \mathrm{kHz}$, which makes it unfeasible to define a threshold to detect incipient damage. Variations in the indices are also observed at frequencies up to $50 \mathrm{kHz}$. Although the variations caused by the vibration at higher frequencies are lower than the variations caused by the damage induced in this study, the detection of less expressive damage may be infeasible.

A similar analysis was performed concerning the effects of the white noise. The results obtained for a noise of $0.2 \mathrm{Vpp}$ are shown in Figure 15. The indices were computed using an impedance signature measured 


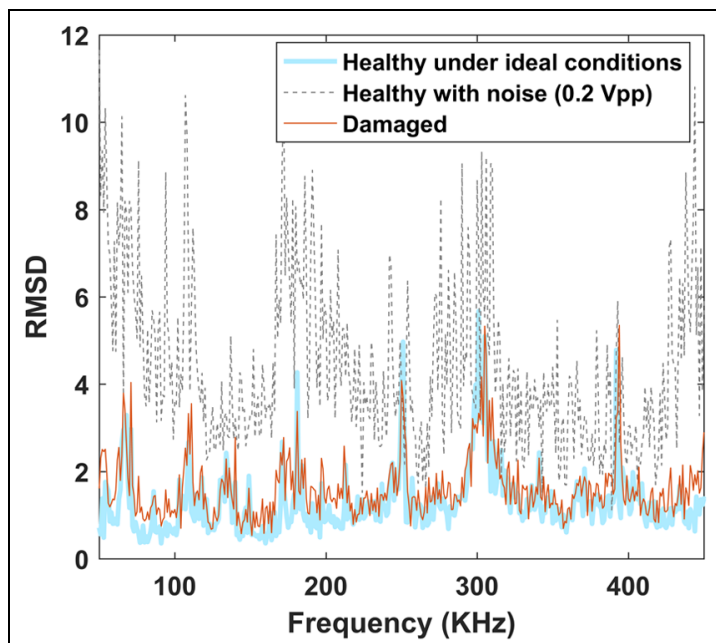

(a)

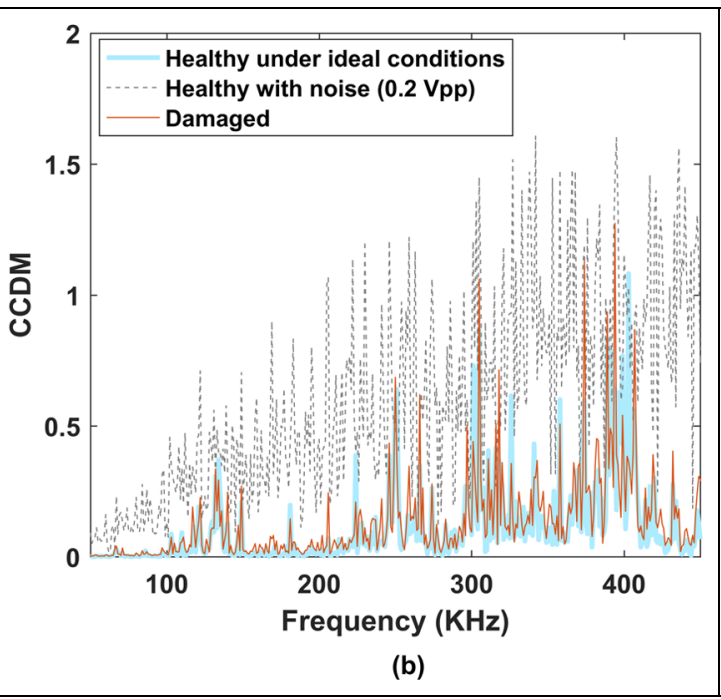

(b)

Figure I5. (a) RMSD and (b) CCDM obtained for ideal conditions, under white noise and with induced damage.

without vibration generation as the baseline, as described above.

As noted in Figure 15, a noise of low intensity causes significant variations in the RMSD and CCDM indices over the entire frequency range. The variations in the indices caused by noise are higher than the variations caused by structural damage. Therefore, the detection of damage is not feasible under this condition, and it is not possible to define a safe threshold for the proper detection of incipient damage.

\section{Compensation for noise effects}

Based on the results presented in the previous sections, external vibration and noise are critical in SHM applications based on the EMI method. Therefore, the compensation for their effects is crucial to the correct diagnosis of the monitored structure. A well-known method reported in the literature ${ }^{32}$ for minimizing the effects of vibration and noise involves increasing the excitation signal of the transducer, which is the $x(t)$ signal indicated in Figure 2.

To analyze the effectiveness of this method, the damage indices were calculated for an excitation signal with amplitude ranging from 0.5 to $10 \mathrm{~V}$ with a step of $0.5 \mathrm{~V}$ and under the effects of white noise, which has shown to be more critical across the entire frequency band. As an example, the RMSD and CCDM indices obtained for the healthy structure under ideal conditions (no noise) and under the influence of noise of $0.2 \mathrm{Vpp}$ are shown in Figure 16. An impedance signature obtained under ideal conditions (i.e. no disturbance) was considered the baseline.

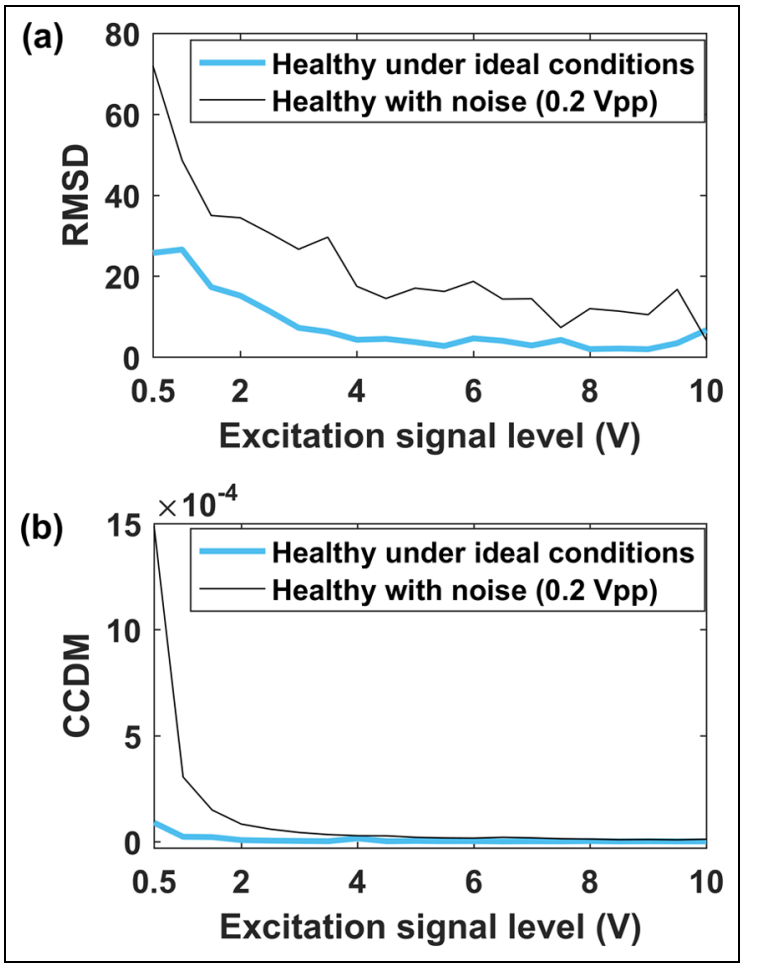

Figure 16. (a) RMSD and (b) CCDM indices obtained for excitation signals of different levels.

The indices in Figure 16 were calculated for the frequency band of $25-50 \mathrm{kHz}$. According to the results, the excitation signal has significant influence on the damage indices in noisy environments. Both indices decrease significantly as the level of the excitation signal increases, converging to the values obtained under ideal conditions. The RMSD index decreased from 71.96 to 4.18 as the excitation signal increased from 0.5 
to $10 \mathrm{~V}$. For the CCDM index, the variation was more pronounced, decreasing from 0.001489 to 0.000012 for the same variation in voltage. Therefore, the results indicate that increasing the excitation signal level is an effective method for compensating the effects of external disturbances.

\section{Conclusion}

In this article, we present an experimental analysis of the effects of external vibration and noise on SHM applications based on the EMI method, which represent a critical problem in the diagnosis of the monitored structure. Tests were performed on an aluminum bar under different levels of vibration and noise to obtain different SNR levels. The experimental results indicate that even a low noise causes significant variations in the impedance signatures and, consequently, in the damage indices, making it difficult to define an appropriate threshold to detect incipient damage. Another important result is that external vibration of low frequency related to rotating devices, which in this study was generated by a chirp signal, can cause variations in the impedance signatures at higher frequencies. As noted in the last section, the effects of external disturbance can be compensated by increasing the excitation signal level of the piezoelectric transducer, provided that the variations in the damage indices are minimized to the same level as the ideal condition. Therefore, the experimental analysis presented in this study can motivate the development of more advanced signal processing techniques and more effective indices for damage detection under conditions of external vibration and noise, which typically occur in real-world applications.

\section{Acknowledgements}

L.M.C. thanks the Capes Foundation, Ministry of Education of Brazil. The authors are grateful to the anonymous reviewers for their valuable recommendations for improving this article.

\section{Declaration of conflicting interests}

The author(s) declared no potential conflicts of interest with respect to the research, authorship, and/or publication of this article.

\section{Funding}

The author(s) disclosed receipt of the following financial support for the research, authorship, and/or publication of this article: This work was funded by the São Paulo Research Foundation (Fapesp) under grant no. 2015/02500-6.

\section{References}

1. Farrar CR and Worden K. An introduction to structural health monitoring. Philos T R Soc A 2007; 1851(365): 303-315.

2. Farrar CR and Worden K. Structural health monitoring: a machine learning perspective. Chichester: John Wiley \& Sons, 2013.

3. Mangalgiri PD and Harinarayana K. Structural health monitoring. In: Prasad NE and Wanhill RJH (eds) Aerospace materials and material technologies: aerospace material technologies, vol. 2. Singapore: Springer, 2016, pp. 449-477.

4. Chatzi E and Papadimitriou C. Identification methods for structural health monitoring, vol. 567. Cham: Springer, 2016, p. 170.

5. Pearson $\mathrm{MR}$, Eaton $\mathrm{M}$, Featherston $\mathrm{C}$, et al. Improved acoustic emission source location during fatigue and impact events in metallic and composite structures. Struct Health Monit. Epub ahead of print 7 October 2016. DOI: $10.1177 / 1475921716672206$.

6. Gianneo A, Carboni M and Giglio M. Feasibility study of a multi-parameter probability of detection formulation for a Lamb waves-based structural health monitoring approach to light alloy aeronautical plates. Struct Health Monit. 2017; 16(2): 225-249.

7. Siivola JT, Minakuchi S, Mizutani T, et al. Evaluation of damage detectability in practical sandwich structure application conditions using distributed fiber optic sensor. Struct Health Monit 2016; 15(1): 3-20.

8. Giurgiutiu V and Zagrai A. Damage detection in thin plates and aerospace structures with the electromechanical impedance method. Struct Health Monit 2005; 4(2): 99-118.

9. Malinowski P, Wandowski T and Ostachowicz W. The use of electromechanical impedance conductance signatures for detection of weak adhesive bonds of carbon fibre-reinforced polymer. Struct Health Monit 2015; 14(4): 332-344.

10. Rabelo DS, Steffen V Jr, Finzi Neto RM, et al. Impedance-based structural health monitoring and statistical method for threshold-level determination applied to 2024-T3 aluminum panels under varying temperature. Struct Health Monit. Epub ahead of print 7 October 2016. DOI: $10.1177 / 1475921716671038$.

11. ANSI/IEEE 176:1988. IEEE standard on piezoelectricity: an American National Standard.

12. Deraemaeker A, Reynders E, De Roeck G, et al. Vibration-based structural health monitoring using output-only measurements under changing environment. Mech Syst Signal Pr 2008; 22(1): 34-56.

13. Baptista FG, Budoya DE, Almeida VAD, et al. An experimental study on the effect of temperature on piezoelectric sensors for impedance-based structural health monitoring. Sensors 2014; 14(1): 1208-1227.

14. Bastani A, Amindavar H, Shamshirsaz M, et al. Identification of temperature variation and vibration disturbance in impedance-based structural health monitoring using 
piezoelectric sensor array method. Struct Health Monit 2012; 11(3): 305-314.

15. Takahashi Y. Evaluation of environmental noise by analyzing faint random vibration in structural health monitoring. J Acoust Soc Am 2016; 140: 2958.

16. Amezquita-Sanchez JP and Adeli H. Signal processing techniques for vibration-based health monitoring of smart structures. Arch Comput Method E 2016; 23(1): $1-15$.

17. Jung HK, Jo H, Park G, et al. Relative baseline features for impedance-based structural health monitoring. J Intel Mat Syst Str 2014; 25(18): 2294-2304.

18. Kim MK, Kim EJ, An YK, et al. Reference-free impedance-based crack detection in plates. J Sound Vib 2011; 330(24): 5949-5962.

19. Overly TG, Park G, Farinholt KM, et al. Piezoelectric active-sensor diagnostics and validation using instantaneous baseline data. IEEE Sens $J$ 2009; 9(11): 1414-1421.

20. Lu L, Song H, Yuan W, et al. Baseline-free damage identification of metallic sandwich panels with truss core based on vibration characteristics. Struct Health Monit 2017; 16(1): 24-38.

21. Worden K, Farrar CR, Manson G, et al. The fundamental axioms of structural health monitoring. $P R$ Soc $A$ 2007; 463(2082): 1639-1664.

22. Annamdas VGM and Annamdas KK. Different types of piezoceramic-structure interaction models using electro mechanical impedance technique: a review. In: Proceedings of SPIE 7292, sensors and smart structures technologies for civil, mechanical, and aerospace systems, San Diego, CA, 8 March 2009, pp. 1-12. SPIE Digital Library.

23. Liang C, Sun FP and Rogers CA. Coupled electromechanical analysis of adaptive material systemsdetermination of the actuator power consumption and system energy transfer. J Intel Mat Syst Str 1994; 5(1): 12-20.

24. Rugina C, Enciu D and Tudose M. Numerical and experimental study of circular disc electromechanical impedance spectroscopy signature changes due to structural damage and sensor degradation. Struct Health Monit 2015; 14(6): 663-681.

25. Roth W and Giurgiutiu V. Structural health monitoring of an adhesive disbond through electromechanical impedance spectroscopy. Int J Adhes Adhes 2017; 73: 109-117.

26. Kaur N, Bhalla S, Shanker R, et al. Experimental evaluation of miniature impedance chip for structural health monitoring of prototype steel/RC structures. Exp Techniques 2016; 40(3): 981-992.

27. Peairs DM, Inman DJ and Park G. Circuit analysis of impedance-based health monitoring of beams using spectral elements. Struct Health Monit 2007; 6(1): 81-94.

28. Baptista FG and Vieira Filho J. A new impedance measurement system for PZT-based structural health monitoring. IEEE T Instrum Meas 2009; 58(10): 3602-3608.

29. Taylor SG, Raby EY, Farinholt KM, et al. Active-sensing platform for structural health monitoring: development and deployment. Struct Health Monit 2016; 15(4): 413-422.

30. Peterson APG. Handbook of noise measurement. 9th ed. Concord, MA: General Radio Company, 1980, p. 394.

31. Freitas ES and Baptista FG. Experimental analysis of the feasibility of low-cost piezoelectric diaphragms in impedance-based SHM applications. Sensor Actuat A: Phys 2016; 238: 220-228.

32. Park G, Sohn H, Farrar CR, et al. Overview of piezoelectric impedance-based health monitoring and path forward. Shock Vib Digest 2003; 35(6): 451-463. 